\title{
Genetically Determined Differences in Brain Response to a Primary Food Reward
}

\author{
Jennifer A. Felsted, ${ }^{1}$ Xueying Ren, ${ }^{1,2}$ Francois Chouinard-Decorte, ${ }^{1}$ and Dana M. Small ${ }^{1,2}$ \\ ${ }^{1}$ The John B. Pierce Laboratory, New Haven, Connecticut 06519, and 2Department of Psychiatry, Yale University School of Medicine, New Haven, \\ Connecticut 06519
}

Combining genetic and neuroimaging techniques may elucidate the biological underpinnings of individual differences in neurophysiology and potential vulnerabilities to disease. The TaqIA A1 variant is associated with diminished dopamine $\mathrm{D}_{2}$ receptor density, higher body mass, and food reinforcement. It also moderates the relationship between brain response to food and future weight gain. This suggests that the polymorphism is associated with a fundamental difference in the neurophysiology of food that may predispose toward overeating. An alternative possibility is that factors, such as impulsivity, eating style, reward drive, and perception, which may covary with the polymorphism, influence reward coding and eating behavior. To distinguish between these alternatives, we used functional magnetic resonance imaging to measure neural response to the ingestion of palatable and caloric milkshakes in healthy subjects with $(\mathrm{A} 1+; n=13)$ and without $(\mathrm{A} 1-; n=13)$ the TaqIA A1 allele. The groups were selected from a larger group to be matched for linked individual factors such as age, gender, education, body mass index, impulsivity, eating style, and perceptual responses to the milkshake. We demonstrate an interaction between genotype ( $\mathrm{A} 1+$ vs $\mathrm{A} 1-)$ and stimulus (milkshake vs a tasteless/odorless baseline) in the midbrain, thalamus, and orbital frontal cortex; whereas A1 - shows increased responses to milkshake, $\mathrm{A} 1+$ shows decreased responses to milkshake relative to baseline. This interaction occurs despite similar ratings of milkshake pleasantness, intensity, and familiarity. We therefore conclude that there is a specific association between the TaqIA A1 polymorphism and brain response during ingestion of a palatable food.

\section{Introduction}

Combining genetic and neuroimaging techniques may elucidate the biological underpinnings of individual differences in neurophysiology and potential vulnerabilities to disease (Winterer et al., 2005). Of particular interest for obesity is the TaqIA restriction fragment length polymorphism (rs1800497; GenBank accession number NP_848605.1), located on the novel ANKK1 (ankyrin repeat and protein kinase domain-containing protein 1) regulatory gene downstream from the dopamine $\mathrm{D}_{2}$ receptor $\left(\mathrm{DRD}_{2}\right)$ gene (Neville et al., 2004). Healthy individuals who possess the A1 allele (A1+: A1/A1, A1/A2), compared with those who do not (A1-: $\mathrm{A} 2 / \mathrm{A} 2)$, show diminished $\mathrm{DRD}_{2}$ receptor density (Jönsson et al., 1999) and regional glucose metabolism in regions involved in reward processing and receiving dopaminergic innervations (Noble et al., 1997). This genetic trait has been shown to moderate (1) increased likelihood of obesity (Noble et al., 1994), (2) food reinforcement and intake, especially in obese individuals (Epstein et al., 2007), and (3) the relationship between neural response to food and prospective weight gain (Stice et al., 2008). This suggests that the polymorphism directly influ-

Received Nov. 5, 2009; revised Dec. 9, 2009; accepted Jan. 5, 2010.

This work was supported by National Institutes of Health Grant R03 DA022292-01 and a private donation (D.M.S.). We thank Ivan de Araujo and Maria G. Veldhuizen for valuable comments made during the data analysis and preparation of this manuscript.

Correspondence should be addressed to Dr. Dana M. Small, The John B. Pierce Laboratory, 290 Congress Avenue, New Haven, CT 06519. E-mail: dsmall@jbpierce.org.

D01:10.1523/JNEUROSCI.5483-09.2010

Copyright $\odot 2010$ the authors $\quad 0270-6474 / 10 / 302428-05 \$ 15.00 / 0$ ences the neurophysiology of food reward in such a way as to confer increased risk for abnormal eating. Alternative possibilities include (1) differential food reinforcement and brain response to food reflect adaptations associated with overeating and the metabolic and physiological consequences of being overweight and obese and (2) altered behavioral and brain response to food reflect a third variable associated with adiposity and the polymorphism. For example, carriers of the A1 variant show increased personality traits associated with impulsivity (White et al., 2008), as well as impairments in error feedback learning (Klein et al., 2007), both of which may confer risk for maladaptive eating.

Our aim was to test whether the TaqIA polymorphism is associated with differential brain response to food independently of other factors known to influence response to food reward, including body mass index (BMI), addiction, impulsivity, eating style, and food sensation. We reasoned that, if the TaqIA A1 polymorphism is associated with variations in the neurophysiology of feeding and reward processing, then differential responses in regions known to encode food reward, such as the midbrain, striatum, thalamus, and orbital frontal cortex (OFC) (Small et al., 2001), will be differentially responsive in A1+ individuals relative to A1 - despite the fact that the groups are closely matched for other key individual variations.

\section{Materials and Methods}

Subjects

Forty-four healthy, right-handed nonsmokers, taking no daily medication and having no history of loss of consciousness, psychiatric disorder, 
chemosensory impairment, or food allergies, completed the study. Subjects also scored lower than 27 on the Binge Eating Scale (Gormally et al., 1982), indicating they were free from pathological binge eating behavior. Four subjects were excluded for technical issues or excessive movement during the scanning. Of the remaining 40 subjects, 13 carried one or two copies of the A1 allele and were designated as the $\mathrm{A} 1+$ group. Thirteen A1 - subjects were then chosen from the larger pool to be matched for $\mathrm{BMI}\left(\right.$ mean $\pm \mathrm{SEM} ; \mathrm{A} 1+, 28.59 \pm 1.99 ; \mathrm{A} 1-, 27.58 \pm 1.58 ; F_{(1,24)}=$ $0.17 ; p=0.68)$, age $\left(\mathrm{A} 1+, 23.92 \pm 1.68 ; \mathrm{A} 1-, 25.31 \pm 0.83 ; F_{(1,24)}=\right.$ $0.60 ; p=0.45)$, and gender $(\mathrm{A} 1+, 12$ females, 1 male; $\mathrm{A} 1-, 12$ females, 1 male). We also selected subjects aiming to equate eating style and impulsivity but did not have the subject pool to match specifically for all of these factors. Therefore, subjects were selected to be as close as possible, and analyses were then conducted to verify that there were no significant differences. The results of these tests revealed no significant effects of group and are detailed in Results.

\section{Stimuli}

Flavor stimuli included $100 \mathrm{ml}$ of Hershey's Cookies ' $\mathrm{n}$ ' Cream Milkshake diluted with $10 \mathrm{ml}$ of distilled water and $100 \mathrm{ml}$ of Garelick Ultimate Strawberry Milk, plus $5 \mathrm{~g}$ of sucrose and $1 \mathrm{ml}$ of strawberry flavor (Galaxy Flavors). The tasteless baseline solution consisted of $12.5 \mathrm{~mm}$ $\mathrm{KCl}$ and $1.25 \mathrm{~mm} \mathrm{NaHCO}_{3}$ in distilled water. Liquids were delivered as $0.5 \mathrm{ml}$ of solution over $3 \mathrm{~s}$ using a gustometer system described previously (Veldhuizen et al., 2007). In brief, this system consists of computercontrolled syringe pumps connected to a functional magnetic resonance imaging (fMRI)-compatible custom-designed gustatory manifold via 25-foot length of beverage tubing (Saint-Gobain Performance Plastics). The manifold was mounted on the head coil, and the liquids dripped from the manifold stylus onto the tongue.

\section{Experimental procedures}

Subjects participated in one behavioral and fMRI training session and one fMRI scanning session conducted on a separate days. Genotyping procedures are detailed in the supplemental data (available at www. jneurosci.org as supplemental material). All testing occurred between 11:00 A.M. and 1:00 P.M. Subjects were instructed to eat $2 \mathrm{~h}$ before their session but then no food or drink (except water) until their arrival. At the start and finish of each session, subjects rated feelings of hunger using a visual analog scale labeled as follows: left anchor, "I am not hungry at all"; midpoint, "neutral"; and right anchor, "I have never been more hungry".

Behavioral and $f M R I$ training session. Height and weight were measured while subjects were clothed (no shoes or jackets) using a Detecto 439 Mechanical Scale and calculated using U.S. Centers for Disease Control and Prevention standards $\left[\mathrm{BMI}=\right.$ weight $\left.(\mathrm{kg}) / \mathrm{height}^{2}\left(\mathrm{~m}^{2}\right)\right]$. Next, subjects completed a battery of questionnaires to characterize their eating behavior and personality. Personality was measured with the Tridimensional Personality Questionnaire (TPQ) (Cloninger et al., 1991). Reward sensitivity was measured with the Behavioral Inhibition System/ Behavioral Activation System (BIS/BAS) (Carver and White, 1994). Impulsivity was measured with the Barratt Impulsiveness Scale Version 11 (BIS) (Patton et al., 1995). Eating style was measured with the Dutch Eating Behavior Questionnaire (DEBQ) (van Strien et al., 1986), the Three Factor Eating Questionnaire (TFEQ) (Stunkard and Messick, 1985), Power of Food Scale (Lowe et al., 2009), and Binge Eating Scale (Gormally et al., 1982).

Subjects then provided stimulus ratings in the fMRI simulator. Ratings were made by using a button box to move a curser to a desired location along a line on a computer screen. Stimulus intensity was measured using an adapted cross-modal general Labeled Magnitude Scale (Green et al., 1996). A $100 \mathrm{~mm}$ vertical line scale with "barely detectable" labeled at the lower anchor point and the label "strongest imaginable sensation" at the upper anchor point was used to measure intensity. Subjects rated pleasantness using a $20 \mathrm{~mm}$ cross-modal visual analog scale anchored by "most unpleasant sensation ever" at the left anchor point (-10), "neutral" in the center $(0)$, and "most pleasant sensation ever" at the right anchor point (10). Ratings of familiarity, edibility, and wanting to eat were also measured using $20 \mathrm{~mm}$ visual analog scales, with the following respective labels: at the left anchor, "not familiar at all", "not edible at all", and "I would never want to eat this"; at the middle point, "neutral"; and at the right anchor, "very familiar", "very edible", and "I would want to eat this more than anything". After completing the ratings, subjects underwent a single mock run, which served to familiarize them with the procedure. During the mock and real runs, either a milkshake or tasteless solution (in random order) was presented to the subject for $3 \mathrm{~s}$. After a 1-7 s jitter, the milkshake was followed by a $3 \mathrm{~s}$ tasteless rinse and finally a $4-8$ or $11 \mathrm{~s}$ jitter before the onset of the next event. After delivery of the tasteless solution, the next event would begin between 4 and $8 \mathrm{~s}$ or $11 \mathrm{~s}$. The tasteless event was not followed by a rinse. Subjects were instructed to hold the solution in their mouths and then swallow during the jitter. Several longer jitters were included to aid deconvolution. This paradigm was presented for two blocked runs, each with a duration of 4 min $21 \mathrm{~s}$, and consisted of 20 total presentations for both the milkshake and tasteless event types. After the mock run, subjects were asked to provide a second set of ratings.

fMRI session. Imaging data were acquired with a Siemens $3 \mathrm{~T}$ Trio magnetom scanner at Yale University Magnetic Resonance Research Center, using parameters similar to previous studies identifying neural correlates associated with chemosensation of food (Small et al., 2008).

A high-resolution T1-weighted three-dimensional anatomical image was acquired for each subject with the following parameters: repetition time (TR), $2530 \mathrm{~ms}$; echo time (TE), $3.66 \mathrm{~ms}$; flip angle, $7^{\circ}$; matrix, $256 \times$ 256, 1-mm-thick slices; field of view (FOV), 256; 176 slices. At the beginning of each functional run, the MR signal was allowed to equilibrate over six scans for a total of $12 \mathrm{~s}$, which were subsequently excluded from analysis. Echoplanar imaging was used to image the blood oxygenation level-dependent (BOLD) signal, indicative of regional brain activity, with the following parameters: TR, $2000 \mathrm{~ms}$; TE, $20 \mathrm{~ms}$; flip angle, $80^{\circ}$; FOV, $220 \mathrm{~mm}$; matrix, $64 \times 64$; slice thickness, $3 \mathrm{~mm}$; acquisition of 40 contiguous slices. Slices were acquired in an interleaved method to reduce the crosstalk of the slice selection pulse.

Data were analyzed using SPM5 software (Statistical Parametric Mapping; Wellcome Department of Imaging Neuroscience, London, UK) in MATLAB 7.3.0 (MathWorks). Functional images were time-acquisition corrected to the slice obtained at $50 \%$ of the TR and realigned to the mean image. Anatomical and functional images were normalized to the standard Montreal Neurological Institute template brain implemented in SPM5, resulting in voxel sizes of 3 and $1 \mathrm{~mm}^{3}$, respectively. Functional time-series data was detrended, a method that eliminates at each voxel any linear component matching the global signal (Macey et al., 2004). Functional images were smoothed using a $6 \mathrm{~mm}$ full-width at halfmaximum isotropic Gaussian kernel. Sessions in which head movement during scanning exceeded a predetermined limit of $1 \mathrm{~mm}$ in any direction were excluded.

A design matrix was created at the individual subject level, which identified the onset and duration of each event. Event onsets were defined as the $3 \mathrm{~s}$ time of taste delivery (when solution entered the subject's mouth). To measure neural response during ingestion (consummatory phase of reward), event durations were defined as the $3 \mathrm{~s}$ taste delivery plus the jitter that followed. Milkshake and tasteless presentations were modeled as events of interest, whereas the rinse was modeled as an event of no interest. According to SPM5 convention, a 128 high-pass filter was applied to the time-series data with the aim to remove low-frequency noise and slow signal drifts. General linear model was used to estimate, at each voxel, condition-specific effects. A canonical hemodynamic response function, including a temporal derivative, was used to model neural response to events.

To assess group effects, the parameter estimate images for the conditions for each subject were entered into $2 \times 2$ stimulus (milkshake and tasteless) by group (A1 - and A1+) ANOVA in SPM 5. T-map threshold was set at $p_{\text {uncorrected }}=0.001$ and a 3 -voxel cluster size. Unpredicted responses were considered significant at $p<0.05$ false discovery ratio (FDR) corrected across the entire brain for multiple comparisons. For predicted responses, WFU PickAtlas (The Functional MRI Laboratory, Wake Forest University School of Medicine, Winston-Salem, NC) was used to perform region of interest (ROI) analyses, and peaks were considered significant at $p<0.05 \mathrm{FDR}$ corrected across the total number of voxels across all ROIs (Maldjian et al., 2003). ROIs were dopamine 

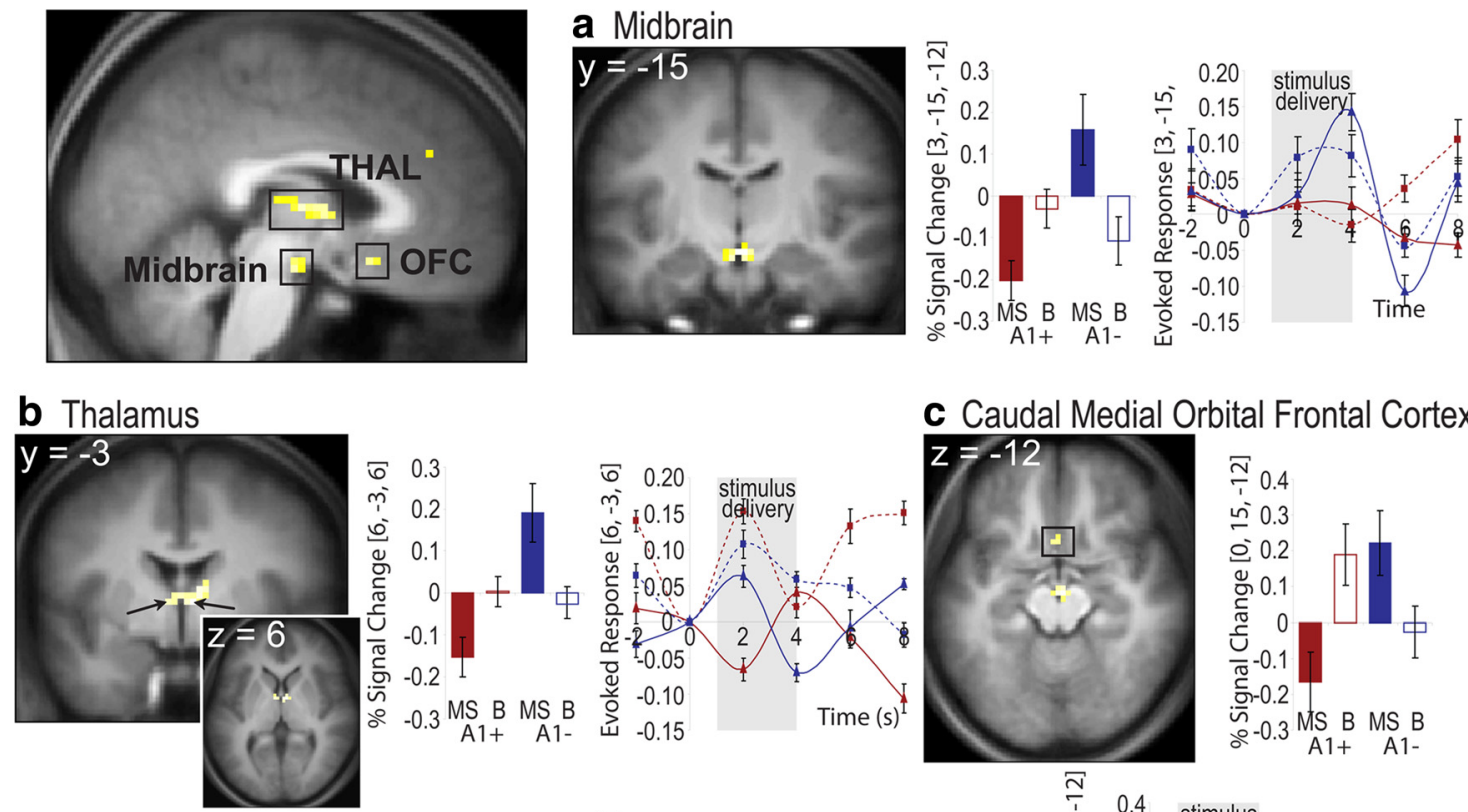

\section{c Caudal Medial Orbital Frontal Cortex}
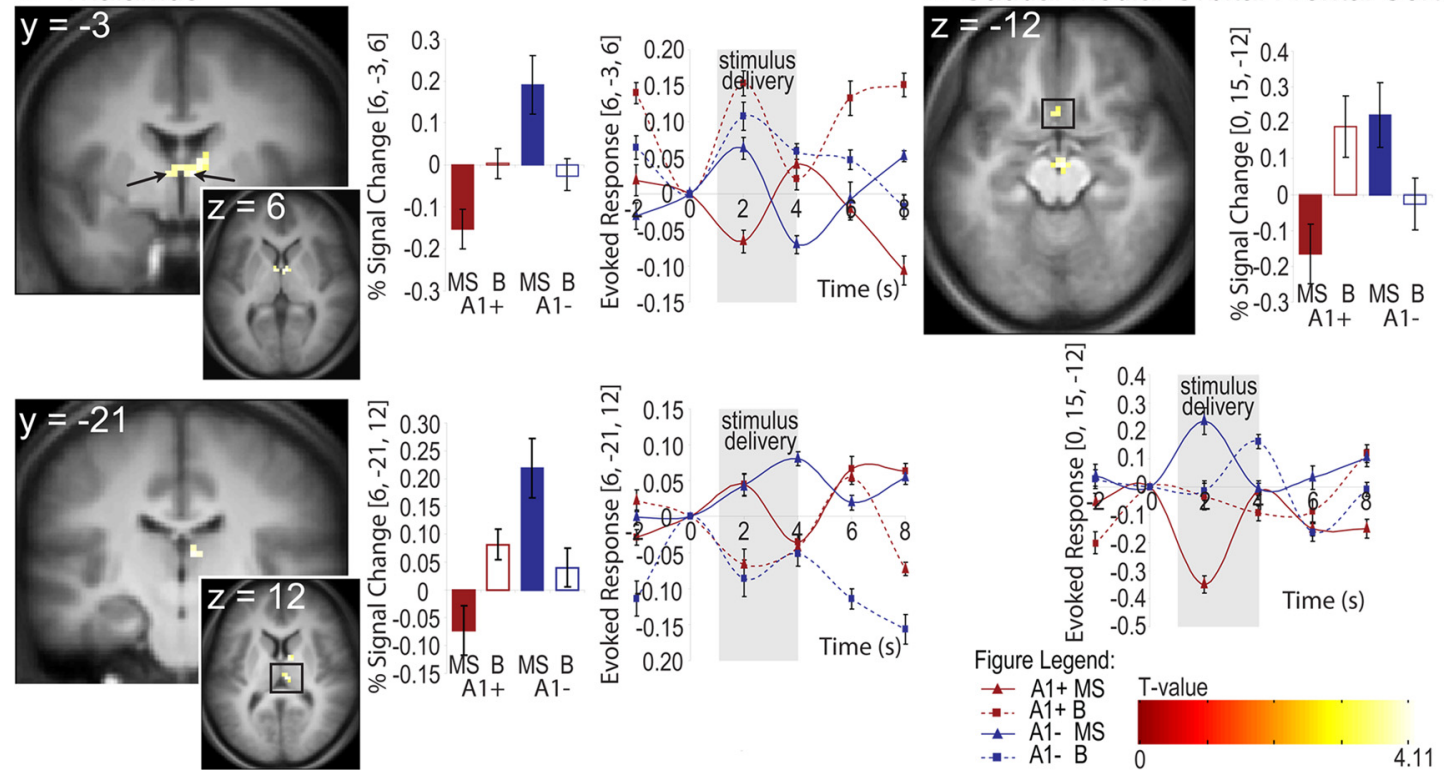

Figure 1. Significant decreased neural responses to milkshake versus tasteless in A1 + versus $\mathrm{A} 1$ - . Sagittal section showing significant effects in midbrain, thalamus, and $0 \mathrm{FC}$. $\boldsymbol{a}$, Coronal section depicting midbrain $([3,-15,-12], t=4.11, p<0.05$, FDR corrected). $\boldsymbol{b}$, Coronal and axial sections depicting anterior nucleus of the thalamus (top) $([-6,-3,6], t=3.71, p<0.05, \mathrm{FDR}$ corrected; $[6,-3,6], t=4.05, p<0.05$, FDR corrected; mediodorsal thalamus, $[6,-21,12], t=3.97, p<0.05$, FDR corrected). A third significant thalamic activation in the mediodorsal thalamus was observed at $[-3,-12,9](t=3.82, p<0.05$, FDR corrected) but is not depicted. c, Axial section depicted OFC $([0,15,-12], t=3.51, p<0.05$, FDR corrected). All bar graphs show percentage \pm SEM signal change extracted from the respective region for each condition (averaged across subjects for each group). Line graphs display the time course of the evoked signal for each condition ( \pm SEM, averaged across subjects for each group), with gray panels illustrating 3 s stimulus delivery. MS, Milkshake; $B$, tasteless.

source and target regions that are also known to play a role in food reward, including the midbrain, striatum, thalamus, OFC, insula, and amygdala (Small et al., 2001; Sánchez-González et al., 2005; GarcíaCabezas et al., 2007, 2009).

\section{Results}

\section{Questionnaires and rating scales}

Sensitivity to reward, impulsivity, and eating style were similar between the groups (reward sensitivity: BIS/BAS, BIS, $p=0.85$ and BAS total, $p=0.17$; impulsivity: BIS total, $p=0.55$; eating style: DEBQ emotional eating total, $p=0.85$ and diffuse emotions, $p=1.0$; clearly labeled emotions, $p=0.78$; external eating, $p=0.72$; restrained eating, $p=0.38$; TFEQ restraint, $p=0.57$; disinhibition, $p=0.39$; hunger, $p=0.55$; Power of Food Scale food available, $p=0.85$; food present, $p=0.93$; food tasted, $p=$ 0.96 ) (for means, variances, and full statistics for all questionnaires and subscales, see supplemental Table 1, available at www. jneurosci.org as supplemental material). Average hunger ratings were near neutral before and after scanning for both groups (mean \pm SEM before scan: A $1+, 0.63 \pm 1.38$; A1,$- 1.68 \pm 1.09$; mean \pm SEM after scan: A $1+, 1.09 \pm 1.39 ; \mathrm{A} 1-, 2.01 \pm 1.14)$ and did not differ as a function of time $(p=0.53)$ or group $(p=$ $0.54)$, and there were no group $\times$ time interactions $(p=0.91)$.
Before and after scanning, the milkshake was rated as pleasant (before scan: $\mathrm{A} 1+, 5.92 \pm 0.64 ; \mathrm{A} 1-, 5.14 \pm 1.02$; after scan: $\mathrm{A} 1+, 6.03 \pm 0.62 ; \mathrm{A} 1-, 3.77 \pm 1.10$ ), edible (before scan: $\mathrm{A} 1+$, $8.35 \pm 0.42$; A1,$- 7.65 \pm 0.73$; after scan: $\mathrm{A} 1+, 7.83 \pm 0.58$; $\mathrm{A} 1-, 7.22 \pm 0.59$ ), wanted (before scan: $\mathrm{A} 1+, 5.86 \pm 0.99$; $\mathrm{A} 1-$, $3.27 \pm 1.55$; after scan: $\mathrm{A} 1+, 4.26 \pm 1.22$; $\mathrm{A} 1-, 3.18 \pm 1.45)$, familiar (before scan: $\mathrm{A} 1+, 8.58 \pm 0.26$; A1,$- 8.31 \pm 0.52$; after scan: $\mathrm{A} 1+, 8.76 \pm 0.26 ; \mathrm{A} 1-, 8.78 \pm 0.30)$, and moderately intense (before scan: $\mathrm{A} 1+, 38.54 \pm 4.9$; $\mathrm{A} 1-, 42.38 \pm 6.7$; after scan: $\mathrm{A} 1+, 36.46 \pm 5.0 ; \mathrm{A} 1-, 42.54 \pm 6.6)$ by both groups with no significant effects of time (pleasant, $p=0.27$; edibility, $p=$ 0.13 ; wanting, $p=0.18$; familiarity, $p=0.21$; intensity, $p=0.69$ ), group (pleasant, $p=0.16$; edibility, $p=0.39$; wanting, $p=0.29$; familiarity, $p=0.76$; intensity, $p=0.53$ ) or group $\times$ time interaction (pleasant, $p=0.20$; edibility, $p=0.88$; wanting, $p=0.22$; familiarity, $p=0.32$; intensity, $p=0.22$ ). The only significant effect of group that was observed was on reward drive 1 from the TPQ ( $p=0.04)$ (supplemental Table 1, available at www.jneurosci. org as supplemental material), with $\mathrm{A} 1+$ having a lower score than A1-; however, this peak did not survive correction for multiple subscale factors within that questionnaire. In summary, the groups were well matched for age, gender, impulsivity, perceptual ratings of 
the milkshake, and internal state at the time of scanning, but the $\mathrm{A} 1$ - subjects showed a trend toward greater reward drive compared with $\mathrm{A} 1+$.

\section{Imaging data}

A main effect of stimulus (milkshake vs tasteless) was observed in the gustatory network (taste thalamus, insula, and operculum) and striatum (putamen and globus pallidus), extending into the amygdala (supplemental Table 2, available at www.jneurosci.org as supplemental material). More importantly, a significant interaction between genotype and stimulus was found in the midbrain (likely corresponding to the substantia nigra), thalamus (anterior thalamic and mediodorsal nuclei), and the caudal medial OFC (Fig. 1). Inspection of the within-subject contrasts revealed that the interaction reflected greater responses in the thalamus and midbrain to milkshake versus tasteless in the A1 - group and a trend toward the opposite pattern (reduced response in milkshake vs tasteless) in the A1+ group (supplemental Tables 3, 4, available at www.jneurosci.org as supplemental material). The effect in the OFC was driven by the attenuated response to milkshake versus tasteless in the A1+ group. These relationships can be observed in the graphs in Figure 1. At a lower threshold, similar effects were observed in the caudate $([9,0,12] ; z=3.6 ; p=$ $0.06)$ and hippocampus ( $[-33,-12,-15] ; z=3.2 ; p=0.1)$, consistent with previous findings (Klein et al., 2007; Stice et al., 2008). No significant effects were observed in the opposite direction (e.g., A1+ greater than A1). Because we observed a significant group difference on the reward drive subscale of the TPQ, we included this score as a covariate of no interest and reran our imaging analysis. This procedure had no effect on the results ( $t$ values differed by less than \pm 0.2 for all areas). In addition, we extracted the contrast estimates from the peak voxel for each area and correlated these values with score on the reward drive 1 factor. Consistent with the analysis of covariance, no significant correlations resulted [range, $r=0.04$ (midbrain) to $r=0.37$ (OFC) $]$. We do note that the $p$ value for the OFC was 0.07 before correction for multiple comparisons, indicating that reward drive cannot be ruled out as contributing to the differential orbital response.

\section{Discussion}

The Al variant of the TaqIA polymorphism is associated with diminished $\mathrm{DRD}_{2}$ receptor density (Jönsson et al., 1999), as well as a number of impulse control disorders, including addiction and obesity (Comings and Blum, 2000) This suggests that the polymorphism is associated with fundamental differences in the neurophysiology of primary reward. With respect to obesity, carriers of the A1 allele show increased food reinforcement (Epstein et al., 2007) and a greater likelihood for obesity (Noble et al., 1994). The polymorphism also moderates the relationship between brain response to food and future weight gain (Stice et al., 2008). This suggests that the polymorphism is associated with a fundamental difference in the neurophysiology of food that may predispose toward overeating.

Consistent with this possibility, although both groups showed the typical responses to milkshake versus tasteless in the gustatory network and ventral striatum, we observed opposing patterns of brain response to milkshake versus tasteless in the nigrothalamo-prefrontal circuit as a function of genotype (Fig. 1). Whereas the A1 - group showed a significantly increased response in this circuit to milkshake compared with tasteless, the $\mathrm{A} 1+$ group tended to show a decreased response to the milkshake relative to the tasteless solution. Critically, we carefully selected the groups from a larger pool of subjects to be matched, or approximately equivalent for, factors, such as impulsivity, eating style, reward drive, and perception, which may covary with the polymorphism, influence reward coding, and/or eating behavior. Therefore, we can conclude that it is the genotype itself, rather than factors linked with the genotype, that resulted in opposing brain response to a primary reward. One caveat to this statement is that $\mathrm{A} 1+$ carriers tended to have lower scores on reward drive, and, although including reward drive as a covariate did not change the OFC $t$ value, the magnitude of the differential response in this region did trend toward being correlated with reward drive score.

Although differential BOLD response does not provide direct evidence for altered dopaminergic activity, we note that the effect we observed was confined to regions that are the source or targets of dopamine neurons, which in primates includes mediodorsal thalamus (Ilinsky et al., 1985; Schultz et al., 1997; SánchezGonzález et al., 2005; Volkow et al., 2008). The possibility that our BOLD effect is linked to the dopamine system is consistent with the known association between reduced $\mathrm{D}_{2}$ receptor density in A1 allele carriers (Noble et al., 1991) and with a recent study showing reduced brain volume in the substantia nigra, which contains dopamine cell bodies, in carriers versus noncarriers of the A1 allele (Cerasa et al., 2009). Given the possible association with the dopamine system and the fact that our milkshake and tasteless deliveries were random, an alternative interpretation of our result that is worthy of follow up is that the interaction we observed may reflect differences in reward prediction rather than in response to the reward itself.

Future work will also be important to determine the behavioral or physiological correlates of the observed effect of genotype on brain response to milkshake and to examine whether gender or BMI influence the interaction between brain coding of food and genotype. Here, 24 of 26 subjects were women, and most were overweight. This leaves open the possibility that our finding is specific to overweight women.

\section{References}

Carver CS, White TL (1994) Behavioral inhibition, behavioral activation, and affective responses to impending reward and punishment: the BIS/ BAS scales. J Pers Soc Psychol 67:319-333.

Cerasa A, Gioia MC, Tarantino P, Labate A, Arabia G, Annesi G, Lanza P, Di Palma G, Blasi V, Quattrone A (2009) The DRD2 TaqIA polymorphism associated with changed midbrain volumes in healthy individuals. Genes Brain Behav 8:459-463.

Cloninger CR, Przybeck TR, Svrakic DM (1991) The tridimensional personality questionnaire: U.S. normative data. Psychol Rep 69:1047-1057.

Comings DE, Blum K (2000) Reward deficiency syndrome: genetic aspects of behavioral disorders. Prog Brain Res 126:325-341.

Epstein LH, Temple JL, Neaderhiser BJ, Salis RJ, Erbe RW, Leddy JJ (2007) Food reinforcement, the dopamine D2 receptor genotype, and energy intake in obese and nonobese humans. Behav Neurosci 121:877-886.

García-Cabezas MA, Rico B, Sánchez-González MA, Cavada C (2007) Distribution of the dopamine innervation in the macaque and human thalamus. Neuroimage 34:965-984.

García-Cabezas MA, Martínez-Sánchez P, Sánchez-González MA, Garzón M, Cavada C (2009) Dopamine innervation in the thalamus: monkey versus rat. Cereb Cortex 19:424-434.

Gormally J, Black S, Daston S, Rardin D (1982) The assessment of binge eating severity among obese persons. Addict Behav 7:47-55.

Green BG, Dalton P, Cowart B, Shaffer G, Rankin K, Higgins J (1996) Evaluating the "Labeled Magnitude Scale" for measuring sensations of taste and smell. Chem Senses 21:323-334.

Ilinsky IA, Jouandet ML, Goldman-Rakic PS (1985) Organization of the nigrothalamocortical system in the rhesus monkey. J Comp Neurol 236:315-330.

Jönsson EG, Nöthen MM, Grünhage F, Farde L, Nakashima Y, Propping P, 
Sedvall GC (1999) Polymorphisms in the dopamine D2 receptor gene and their relationships to striatal dopamine receptor density of healthy volunteers. Mol Psychiatry 4:290-296.

Klein TA, Neumann J, Reuter M, Hennig J, von Cramon DY, Ullsperger M (2007) Genetically determined differences in learning from errors. Science 318:1642-1645.

Lowe MR, Butryn ML, Didie ER, Annunziato RA, Thomas JG, Crerand CE, Ochner CN, Coletta MC, Bellace D, Wallaert M, Halford J (2009) The Power of Food Scale. A new measure of the psychological influence of the food environment. Appetite 53:114-118.

Macey PM, Macey KE, Kumar R, Harper RM (2004) A method for removal of global effects from fMRI time series. Neuroimage 22:360-366.

Maldjian JA, Laurienti PJ, Kraft RA, Burdette JH (2003) An automated method for neuroanatomic and cytoarchitectonic atlas-based interrogation of fMRI data sets. Neuroimage 19:1233-1239.

Neville MJ, Johnstone EC, Walton RT (2004) Identification and characterization of ANKK1: a novel kinase gene closely linked to DRD2 on chromosome band 11q23.1. Hum Mutat 23:540-545.

Noble EP, Blum K, Ritchie T, Montgomery A, Sheridan PJ (1991) Allelic association of the D2 dopamine receptor gene with receptor-binding characteristics in alcoholism. Arch Gen Psych 48:648-654.

Noble EP, Noble RE, Ritchie T, Syndulko K, Bohlman MC, Noble LA, Zhang Y, Sparkes RS, Grandy DK (1994) D2 dopamine receptor gene and obesity. Int J Eat Disord 15:205-217.

Noble EP, Gottschalk LA, Fallon JH, Ritchie TL, Wu JC (1997) D2 dopamine receptor polymorphism and brain regional glucose metabolism. Am J Med Genet 74:162-166.

Patton JH, Stanford MS, Barratt ES (1995) Factor structure of the Barratt Impulsivneness Scale. J Clin Psychol 51:768-774.

Sánchez-González MA, García-Cabezas MA, Rico B, Cavada C (2005) The primate thalamus is a key target for brain dopamine. J Neurosci 25: $6076-6083$.

Schultz W, Dayan P, Montague PR (1997) A neural substrate of prediction and reward. Science 275:1593-1599.

Small DM, Zatorre RJ, Dagher A, Evans AC, Jones-Gotman M (2001) Changes in brain activity related to eating chocolate: from pleasure to aversion. Brain 124:1720-1733.

Small DM, Veldhuizen MG, Felsted J, Mak YE, McGlone F (2008) Separable substrates for anticipatory and consummatory food chemosensation. Neuron 57:786-797.

Stice E, Spoor S, Bohon C, Small DM (2008) Relation between obesity and blunted striatal response to food is moderated by TaqIA Al allele. Science 322:449-452.

Stunkard AJ, Messick S (1985) The three-factor eating questionaire to measure dietary restraint, disinhibition and hunger. J Psychosom Res 29:71-83.

van Strien T, Fijters JER, Bergers GPA, Defares P (1986) The Dutch Eating Behavior Questionnaire (DEBQ) for assessment of restrained, emotional, and external eating. Int J Eat Disord 5:295-315.

Veldhuizen MG, Bender G, Constable RT, Small DM (2007) Trying to detect taste in a tasteless solution: modulation of early gustatory cortex by attention to taste. Chem Senses 32:569-581.

Volkow ND, Wang GJ, Fowler JS, Telang F (2008) Overlapping neuronal circuits in addiction and obesity: evidence of systems pathology. Philos Trans R Soc Lond B Biol Sci 363:3191-3200.

White MJ, Morris CP, Lawford BR, Young RM (2008) Behavioral phenotypes of impulsivity related to the ANKK1 gene are independent of an acute stressor. Behav Brain Funct 4:54.

Winterer G, Hariri AR, Goldman D, Weinberger DR (2005) Neuroimaging and human genetics. Int Rev Neurobiol 67:325-383. 